\title{
High-resolution and accelerated multi- parametric mapping with automated characterization of vessel disease using intravascular MRI
}

\author{
Guan Wang ${ }^{1,2}$, Yi Zhang ${ }^{2}$, Shashank Sathyanarayana Hegde ${ }^{2}$ and Paul A. Bottomley ${ }^{1,2^{*}}$
}

\begin{abstract}
Background: Atherosclerosis is prevalent in cardiovascular disease, but present imaging modalities have limited capabilities for characterizing lesion stage, progression and response to intervention. This study tests whether intravascular magnetic resonance imaging (IVMRI) measures of relaxation times $\left(T_{1}, T_{2}\right)$ and proton density (PD) in a clinical 3 Tesla scanner could characterize vessel disease, and evaluates a practical strategy for accelerated quantification.

Methods: IVMRI was performed in fresh human artery segments and swine vessels in vivo, using fast multi-parametric sequences, 1-2 mm diameter loopless antennae and 200-300 $\mu \mathrm{m}$ resolution. $T_{1}, T_{2}$ and PD data were used to train a machine learning classifier (support vector machine, SVM) to automatically classify normal vessel, and early or advanced disease, using histology for validation. Disease identification using the SVM was tested with receiver operating characteristic curves. To expedite acquisition of $T_{1}, T_{2}$ and PD data for vessel characterization, the linear algebraic method ('SLAM') was modified to accommodate the antenna's highly-nonuniform sensitivity, and used to provide average $T_{1}, T_{2}$ and PD measurements from compartments of normal and pathological tissue segmented from high-resolution images at acceleration factors of $R \leq 18$-fold. The results were validated using compartment-average measures derived from the high-resolution scans.
\end{abstract}

Results: The SVM accurately classified $\sim 80 \%$ of samples into the three disease classes. The 'area-under-the-curve' was 0.96 for detecting disease in 248 samples, with $T_{1}$ providing the best discrimination. SLAM $T_{1}, T_{2}$ and PD measures for $R \leq 10$ were indistinguishable from the true means of segmented tissue compartments.

Conclusion: High-resolution IVMRI measures of $\mathrm{T}_{1}, \mathrm{~T}_{2}$ and PD with a trained SVM can automatically classify normal, early and advanced atherosclerosis with high sensitivity and specificity. Replacing relaxometric MRI with SLAM yields good estimates of $T_{1}, T_{2}$ and PD an order-of-magnitude faster to facilitate IVMRI-based characterization of vessel disease.

Keywords: Intravascular MRI, Atherosclerosis, Relaxation times, Disease classification, Accelerated acquisition, SLAM, Machine learning

\footnotetext{
* Correspondence: bottoml@mri.jhu.edu

Dr. Zahi Fayad served as a Guest Editor for this submission.

${ }^{1}$ Department of Electrical \& Computer Engineering, Johns Hopkins University,

Baltimore, MD, USA

${ }^{2}$ Division of MR Research, Department of Radiology and Radiological

Sciences, Johns Hopkins University, Park building 310, 600 N Wolfe Street,

Baltimore, MD 21287, USA
} 


\section{Background}

Atherosclerosis is the prevalent factor in patients presenting with cardiovascular disease, which is responsible for $\sim 1 / 3$ of all deaths in the United States and includes heart disease, stroke, peripheral artery disease, and hypertension [1]. Because plaque evolution from earlystage lesions to those vulnerable to rupture can take decades, differentiation of lesion stage is crucial for assessing progression and measuring the efficacy of dietary, pharmaceutical and intravascular interventions. The American Heart Association (AHA) has classified atherosclerotic lesions as Types I-VI based on the presence of foam cells; extra-cellular lipids; fibrous caps that have a high risk of rupture and causing stroke or myocardial infarction; calcification; fibrosis, and complex ruptured thrombotic lesions [2].

Clinically, atherosclerosis is diagnosed by the presence of significant luminal narrowing using X-ray angiography [1]. However, because X-ray cannot detect vessel wall, non-calciferous lesion morphology or early-stage lesions with mild stenosis [3], it is insensitive to most of the AHA metrics that define disease stage. While both intravascular ultrasound (IVUS) and optical coherence tomography (OCT) can provide transluminal imaging [4], they also require $\mathrm{X}$-ray guidance and may be confounded by calcification $[5,6]$, or penetration depth and the need for a blood-free environment [4], respectively. Advances in all of these modalities are promising [7], but no single technique is presently suited for minimally-invasive assessment of all of the relevant AHA disease characteristics needed to monitor plaque progression or regression [8] or to document the efficacy of therapy or lifestyle changes.

Although noninvasive angiography of vessel lumens with magnetic resonance imaging (MRI) has only modestly impacted vessel wall imaging, MRI's intrinsic sensitivity to soft tissue in noninvasive spin-lattice $\left(T_{1}\right)$ and spin-spin $\left(\mathrm{T}_{2}\right)$ relaxation-weighted 'black blood' studies has permitted differentiation of healthy vessel from atherosclerosis [9-11]. Indeed, meta-analysis of studies employing combined relaxation, proton density (PD) and/or flow weightings shows that MRI detection of intra-plaque hemorrhage in carotid disease is highly predictive of cerebrovascular events [12]. Nevertheless, with a typical carotid artery $\leq 0.75 \mathrm{~mm}$ thick [13] and MRI's spatial resolution of $\sim 0.4 \mathrm{~mm}[9,10]$, vessel walls may occupy only $1-2$ pixels. While spatial resolution and low signal-to-noise ratio (SNR) represent fundamental limits to accurate quantification, current relaxation-weighted MRI approaches for assessing vessel disease [11, 14, 15] can also confound multi-study comparisons and reproducibility for disease classification.

Unlike relaxation-weighted image intensities, the absolute $\mathrm{T}_{1}, \mathrm{~T}_{2}$ and $\mathrm{PD}$ values reflect intrinsic properties of the biological tissue, and might provide more robust metrics for classifying vessel disease by ameliorating instrumental and sequence effects $[11,16]$. Several studies have reported relaxation times for atherosclerotic tissues [17-22]. However, $\mathrm{T}_{1}$ and $\mathrm{T}_{2}$ measurements in vivo are also limited by long scan times and poor SNR at the spatial resolution needed to image thin vessel walls, which compromise quantification. Consequently, multiparametric criteria for classifying vessel disease have yet to be developed.

Intravascular (IV) MRI coils [23-25], especially those operating at higher magnetic field strengths $\left(B_{0} \geq 3\right.$ Tesla, T) $[25,26]$, could dramatically improve spatial resolution and reduce scan times as a result of an intrinsic, realizable, $B_{O}^{2}$-dependence of their SNR $[27,28]$. Biocompatible $3 \mathrm{~T}$ IVMRI coils have visualized calcification, pathology, accurately measured the thickness of fibrous caps on atherosclerotic plaques down to a native resolution of $80 \mu \mathrm{m}$ in several minutes [29], and have performed 'MRI endoscopy' at 1-2 frames(fr)/s and $300 \mu \mathrm{m}$ resolution [25]. Thus, IVMRI could provide the spatial resolution and SNR needed to characterize vessel disease. However, a practical and efficient strategy for acquiring the relaxometric information, would still be required. Unfortunately, the multi-coil based acceleration techniques used in conventional MRI (sensitivity encoding-'SENSE' and the like) are inapplicable to IVMRI which typically involves but a single detector.

Recently, the 'SLAM' (spectroscopy with linear algebraic modeling) localization method was developed to reduce scan-times for spectroscopy [30-33] and relaxometry [34], by directly encoding tissue 'compartments' using a small subset of the highest-SNR spatial-encoding steps. Acceleration factors of up to $R=120$ have been demonstrated [30, 31]. The compartments are defined as areas of relatively uniform-appearing tissue on regular high-resolution or scout images. These can be segmented, re-segmented and reconstructed post-acquisition from the same undersampled data set, using high-resolution scout images as prior knowledge. SLAM could enable highlyaccelerated compartment-average tissue characterization when the acquisition of high-resolution relaxation and/or PD maps is precluded by scan-time constraints [34]. Unfortunately, the standard SLAM method assumes that the detector phase and coil sensitivity are relatively uniform across segmented compartments [30, 31]. This is rarely true for IVMRI detectors, whose sensitivity is highly non-uniform $[27,28]$. Thus, compensation for detector inhomogeneity would be key to accelerated IVMRI relaxometry using SLAM.

Here we posit that the measurement of $T_{1}, T_{2}$, and PD with high-resolution IVMRI could enable the characterization of atherosclerosis in situ. We further posit that SLAM can be adapted for IVMRI and used to provide accurate, highly-accelerated, measurements of 
compartment-average MRI relaxation times, and thereby enable the characterization of plaque components when high-resolution relaxometry is impractical due to scan-time limitations.

For the first time we present high-resolution IVMRI $\mathrm{T}_{1}, \mathrm{~T}_{2}$, and $\mathrm{PD}$ results from autopsied human specimens and swine in vivo. The data were acquired using two fast MRI relaxometry methods: (i) the 'four flip-angle' (FourFA) method, which uses the theoretical minimum number of steady-state acquisitions [35]; and (ii) the 'mixed turbo spin-echo' (MIX-TSE) method [36]. We then trained a supervised machine learning classifier-a support vector machine (SVM) [37, 38]-to automatically classify the stage of vessel disease from the multi-parametric data using histology as a standard. Finally, we adapted the SLAM method to provide highly-accelerated relaxometry with IVMRI detectors, by incorporating simple phase and amplitude sensitivity corrections into the reconstruction. SLAM was applied in vitro and in vivo to provide compartment-average $T_{1}, T_{2}$ and $P D$ characterization up to 18 -fold faster than the fully-sampled IVMRI acquisitions used for validation.

\section{Methods \\ Subjects}

Fresh human iliac and coronary artery segments $(n=10)$ were harvested from decedents ( $n=6$; age $\geq 60$ yrs) in our Pathology Department with Institutional Review Board approval. MRI was performed shortly after harvest ( $<48 \mathrm{~h}$ ). All decedents had a diagnosis of cardiovascular disease and atherosclerotic lesions were evident at gross examination in six specimens. Additional formalin-fixed specimens (excluded from the classifier training set) were used to test the robustness of the SVM algorithm. Specimens were 1-5 cm long, with 0.5-2.2 cm diameter lumens.

To test and compare relaxometry in vivo with the in vitro results in normal vessels, approval was obtained from our Institutional Animal Care and Use Committee for an in vivo study of a female Yorkshire pig. The animal was sedated with an intramuscular combination of ketamine, xylazine and telazol; induced intravenously with propofol to effect (approximately $4 \mathrm{mg} / \mathrm{kg}$ ); and intubated and maintained with general anesthesia during IVMRI using $1 \%-2 \%$ isoflurane under mechanical ventilation. After MRI, the animal was humanely euthanized and vessel specimens harvested.

Transverse sections of the specimens were stained using hematoxylin and eosin (H\&E; enhances calcification, lipid core and intra-plaque hemorrhage), von Kossa (enhances mineralization), Verhoeff-van Gieson (VVG; enhances elastic fibers) or Movat (enhances constituents of cardiovascular tissue) methods to visualize tissue histology.

\section{High resolution IVMRI}

IVMRI was performed on a Philips $3 \mathrm{~T}$ Achieva clinical scanner (Philips Healthcare, Best, The Netherlands) using in-house loopless antenna IVMRI coils operating as receivers with body coil excitation. During IVMRI, RF heating of the antenna coils was controlled within $1{ }^{\circ} \mathrm{C}$ using decoupling circuitry and tuned connection cables, as verified by phantom studies and examination of in vitro and in vivo specimens described previously $[28,29]$.

In vitro studies of specimens were performed using a $2.2 \mathrm{~mm}$ outer diameter (OD), $400 \mathrm{~mm}$-long semi-rigid copper coaxial cable antenna (UT-85-C, Micro-coax Inc., Pottstown PA), with a distal whip formed by extending the central conductor by $39 \mathrm{~mm}[28,29]$. With the antenna located in the lumen, specimens were mounted at the center of a phantom filled with $3.5 \mathrm{~g} / \mathrm{l}$ saline to mimic the body's electrical properties at the MRI frequency, and scout MRI was performed. The Four-FA method was applied with a sequence repetition period $\mathrm{T} R=651 \mathrm{~ms} ;$ FAs of $\theta_{1-4}=30^{\circ}, 80^{\circ}, 140^{\circ}, 30^{\circ}$; and a three-dimensional (3D) pixel size $=0.2 \times 0.2 \times 1.6 \mathrm{~mm}^{3}$, to acquire four signals, $\mathrm{S}_{1-4}$ [35]. A $10 \mathrm{~ms}, \mathrm{FA}=0^{\circ} \mathrm{B}_{1}$-independent rotation (BIR4) pre-pulse was added for the $\mathrm{S}_{4}$ acquisition [35]. MIX-TSE data were acquired with TSE $=8$ or 16 echoes (denoted MIX-TSE-8 and MIXTSE-16). The MIX-TSE-8 sequence was performed with $\mathrm{TR}_{\mathrm{SE}}=1000 \mathrm{~ms}, \mathrm{TR}_{\mathrm{IR}}=2260 \mathrm{~ms}$, echo time $\mathrm{TE}_{1 / 2}=28.6 / 100 \mathrm{~ms}$, inversion time $\mathrm{TI}=0.5 \mathrm{~s}$, and voxel size $=0.2 \times 0.2 \times 2 \mathrm{~mm}^{3}$ or $0.2 \times 0.2 \times 4 \mathrm{~mm}^{3}$. MIX-TSE-16 was performed with $\mathrm{TR}_{\mathrm{SE}}=760 \mathrm{~ms}$, $\mathrm{TR}_{\mathrm{IR}}=2290 \mathrm{~ms}, \quad \mathrm{TE}_{1 / 2 / 3 / 4}=25 / 65 / 105 / 145 \mathrm{~ms}$, $\mathrm{TI}=0.5 \mathrm{~s}$, and voxel size $=0.3 \times 0.3 \times 5 \mathrm{~mm}^{3}$. The Four-FA and MIX-TSE sequences are depicted in the accompanying Additional file 1 . The 'three-point Dixon method' [39] was used to separately image water and fat components in lesions $(\mathrm{TR}=0.2 \mathrm{~s}$; $\mathrm{TE}=4.6,5.8,6.9 \mathrm{~ms}$; $\mathrm{FA}=55^{\circ} ; 0.27 \times 0.27 \times 3 \mathrm{~mm}^{3}$ voxels $)$.

The in vivo study utilized a $0.8 \mathrm{~mm}$ OD biocompatible super-elastic nitinol coaxial cable with a $42 \mathrm{~mm}$ whip antenna $[25,28,29]$. The coil was advanced via a femoral incision to the inferior vena cava, and MIX-TSE and water/fat images acquired with the identical imaging sequences used in vitro.

\section{Image processing}

For the purpose of quantification, the antenna's location was detected via its intensity/phase singularities [40] and the high resolution IVMRI signal intensity was corrected for its $1 / r$ dependence with distance, $r$, from the antenna, by $r$-scaling as shown in Fig. 1, except for one case where an interpolated signal map from saline was used. The Four-FA multi-parametric maps were computed from $S_{1-4}$ [35], and the MIX-TSE multi-parametric maps computed as prescribed [41]. The PD maps were 

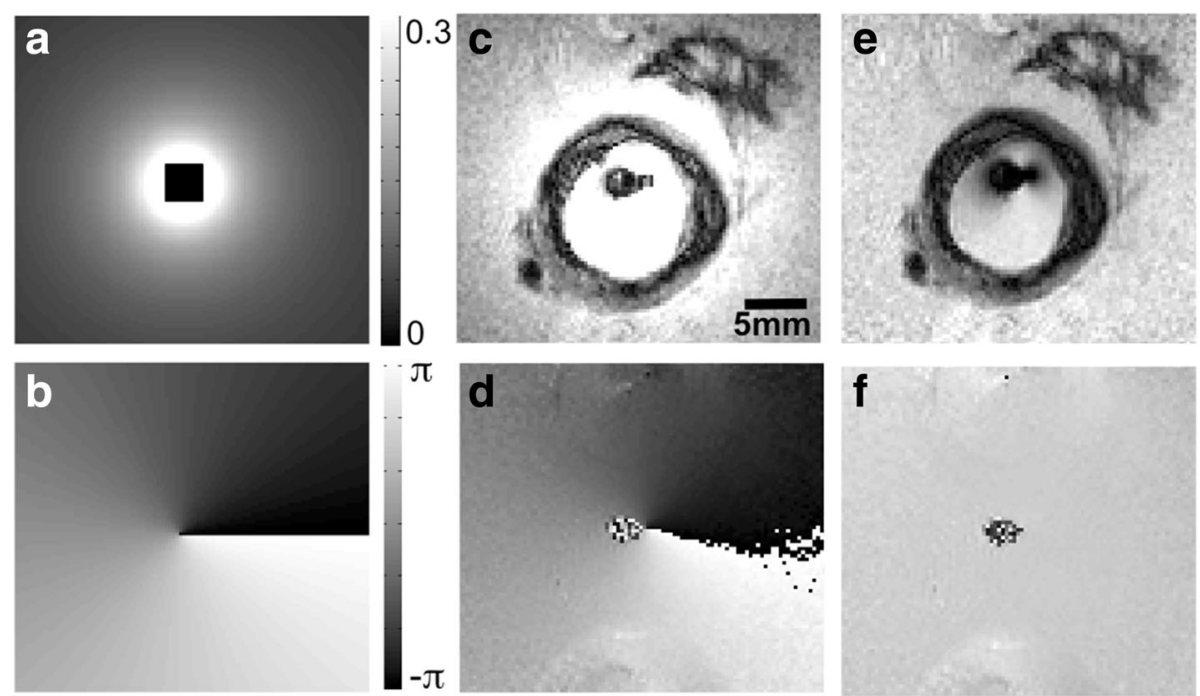

Fig. 1 (a) Simulated 1/r-dependence of the magnitude of the IVMRI signal with radial distance from the antenna (central black square), and (b), the linear $0-2 \pi$ phase map of the MRI signal assumed for high resolution IVMRI image correction and for SLAM reconstruction. (c, e) Magnitude and (d, f) phase $200 \mu \mathrm{m}$ resolution MIX-TSE images of a diseased vessel from a myelodysplastic patient depicted without (c, d) and with (e, $\mathbf{f}$ ) these corrections. The phase scale in (b) also applies to (d) and (f)

normalized relative to a $\mathrm{PD}=1.0$ assumed for water in saline. In vitro and in vivo compartment-average $\mathrm{T}_{1}$, $\mathrm{T}_{2}$ and $\mathrm{PD}$ values were measured in anatomically distinct compartments of relatively uniform-appearing tissue, manually segmented from fully-sampled ( $k$-space) anatomical, intensity-corrected images, and the values averaged.

\section{Tissue classification}

A Python-based (Python 2.7.10 interpreter, sklearn 0.17) linear kernel SVM classifier was trained and validated using $\mathrm{T}_{1}, \mathrm{~T}_{2}$ and $\mathrm{PD}$ values from the conventional reconstructed multi-parametric maps. Because the presence of lipid within (vs. outside) vessel walls was uncommon in Dixon images in our sample set, it was excluded as an independent classifier. Data points $(n=248)$ were randomly sampled in vessel walls in artifact-free regions of the $\mathrm{T}_{1}, \mathrm{~T}_{2}$ and $\mathrm{PD}$ images with adequate SNR $(\geq 5)$. Healthy and diseased tissues in the freshly-harvested specimens were histologically classified as smooth muscle cells (SMC; light pink on H\&E), early stage disease (green/blue on Movat, brown in Von Kossa, purple in VVG) or advanced disease (black on Movat and VVG) on sections that were anatomically coregistered with the multi-parametric maps. The samples were labeled with the histology result, and the corresponding $\mathrm{T}_{1}, \mathrm{~T}_{2}$, and PD values. The SVM was trained using the samples and validated using the 'leave-one-out' [42] method. The trained SVM was also tested on the formalin-fixed specimen for robustness. The three-class SVM classifier generated probabilities for each sample belonging to each class. The class exhibiting the highest probability was designated as the output class.

A receiver operating characteristic (ROC) [43] analysis was performed to assess the potential sensitivity and specificity of IVMRI-based disease classification. For this purpose, samples were regrouped into disease and healthy SMC classes and used to train a two-class SVM classifier. The discrimination threshold was adjusted from 0 to 1 on the output disease probability to generate the ROC curve. To compare the performance of disease classification based on a single MRI parameter, the three-class and twoclass SVMs were re-trained and re-tested using only one of $\mathrm{T}_{1}, \mathrm{~T}_{2}$ or PD as the sample feature.

\section{SLAM IVMRI}

SLAM was applied with the MIX-TSE sequence by selecting all of the high-SNR raw central $k$-space data corresponding to acceleration factors of $R \leq 18$, i.e. by discarding up to $94 \%$ of the outer $k$-space data. Compartments that included all signal sources were segmented from the full $k$-space images of the same sections. The latter served as 'prior knowledge' for generating highresolution masks for reconstructing the compartmentaverage signals [30, 31] (see Additional file 1). Because the magnitude and phase of the antenna's signal exhibited strong gradients across anatomical compartments, the signal was corrected for amplitude and phase variations assuming the same $1 / r$-magnitude and linear $0-2 \pi$ polar phase map depicted in Fig. 1(a, b) for the high-resolution studies. These two factors were incorporated into the SLAM reconstruction as spatially-dependent reciprocals 
of the receiver sensitivity profiles and phase conjugates in the ' $\mathbf{A}_{\mathrm{M}^{*} \mathrm{M}}$ ' matrix in Eq. (10) of Ref. [31]. They were also factored into the discrete spatial response function (dSRF) which defines compartmental localization (see Additional file 1). $T_{1}, T_{2}$ and $P D$ values were then computed from the compartment-average signals using the same relaxation formula used for the full $k$-space MIX-TSE MRI data $[35,41]$.

SLAM $\mathrm{T}_{1}, \mathrm{~T}_{2}$ and PD values were compared with the full $k$-space results averaged over the same compartments at different acceleration factors, to test whether high-speed sensitivity-corrected SLAM could accurately characterize vessel disease components when scan-time for high-resolution relaxometry protocols is limiting.

\section{Results}

The amplitude and phase corrections of Fig. 1(a,b) normalized the high resolution IVMRI signal intensity, as shown in a full $k$-space MIX-TSE-16 image of a vessel specimen from a patient with myelodysplastic syndrome (Fig. 1e, f). Figure 2(a) shows a $200 \mu \mathrm{m}$ resolution IVMRI of a specimen with a plaque and peripheral fat evident in the Dixon lipid image (blue). Movat and Van Kossa staining confirm the presence of early-stage thickening and fibrosis (Fig. 2b-c). Figure $2(\mathrm{~d}-\mathrm{f})$ show the Four-FA $\mathrm{T}_{1}, \mathrm{~T}_{2}$, and PD maps at the same resolution: the saline $T_{1}$ and $T_{2}$ are beyond the validation range [35] and are masked (green) for clarity.

The measured $T_{1}, T_{2}$, and PD values of the randomly sampled points from the full $k$-space images of human vessel specimens are color-coded by tissue class and plotted in Fig. 3. The means \pm SD (standard deviation) summarized in Table 1, are consistent with published $\mathrm{T}_{2}$ values of $54 \pm 13 \mathrm{~ms}$ [17], $76 \pm 9 \mathrm{~ms}$ [21], $69.1 \pm 6.6 \mathrm{~ms}$ [22] and $39 \pm 5 \mathrm{~ms}$ [20]; and $\mathrm{T}_{1}$ values of $685.9 \pm 166 \mathrm{~ms}$ [22] and $844 \pm 96 \mathrm{~ms}$ [20], for normal vessel wall at $3 \mathrm{~T}$. Published $\mathrm{T}_{2}$ values for lipid-rich advanced lesions are $37 \pm 5 \mathrm{~ms}$ [17] and $54 \pm 3 \mathrm{~ms}$ [21].

The results of the 'leave-one-out' cross-validation of the three-class SVM are also included in Table 1. The three-class SVM correctly classified $>83 \%$ of SMC and advanced lesions, and had an overall accuracy of $78 \%$. Figure 4 exemplifies validation in a fresh vessel segment (Fig. 4a) with corresponding VVG-histology (Fig. 4b). When applied to a formalin-fixed vessel segment (Fig. 4c), the classifier correctly differentiated all advanced lesion samples $\left(n=19\right.$; mean $\mathrm{T}_{1}=390 \pm 90 \mathrm{~ms}$, $\mathrm{T}_{2}=22 \pm 6 \mathrm{~ms}$ and $\mathrm{PD}=0.35 \pm 0.12$ ) from SMC $\left(n=13 ; \mathrm{T}_{1}=873 \pm 144 \mathrm{~ms} ; \mathrm{T}_{2}=64 \pm 11 \mathrm{~ms} ;\right.$ $\mathrm{PD}=0.64 \pm 0.06$ ), as identified by histology (Fig. $4 \mathrm{~d}$ ).

The two-class SVM yielded an ROC curve for distinguishing disease from non-disease with the highest overall accuracy and area under curve (AUC) of 0.96 using all three parameters (Fig. 5a). With the three parameters tested separately, $\mathrm{T}_{1}$ afforded the highest sensitivity and specificity for differentiating vessel disease from healthy tissue, with an AUC $=0.94$, that accounted for nearly all of the three parameter result (Fig. 5b-d). By itself, PD was least able to discriminate disease (AUC $=0.6$ ). The two-class SVM correctly classified the SLAM data as well, including vena cava, in vivo.

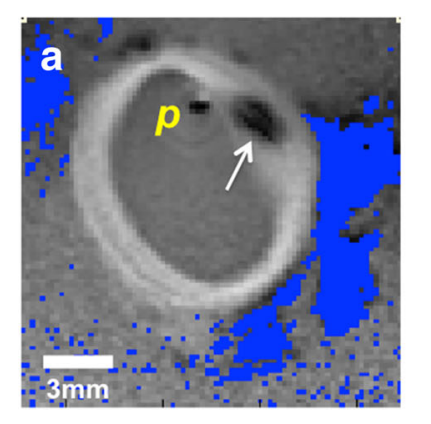

b
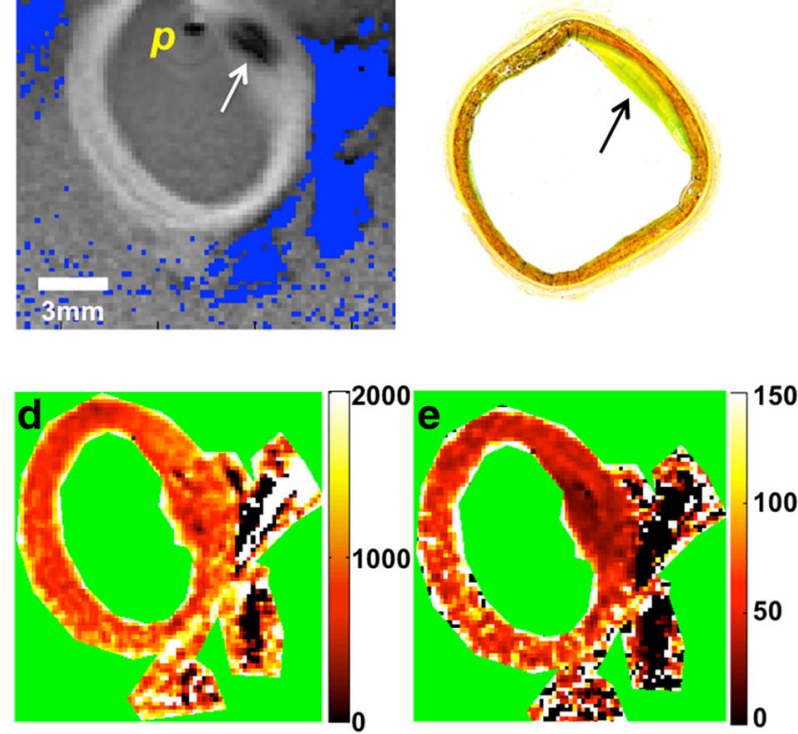

C
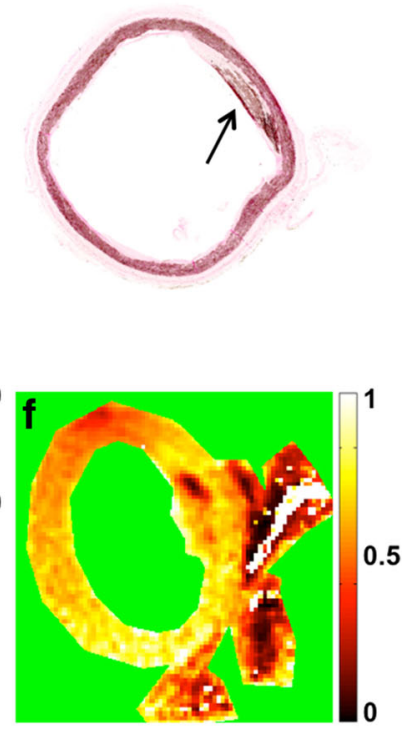

Fig. 2 (a) Dixon lipid image (blue) overlaid on a gradient-echo vessel wall image, with (b) Movat and (c) Van Kossa histology results from the vessel section post-exam (arrows, calcified lesion; ' $p$ ' is near the probe location). Color-coded $T_{1}(\mathbf{d}), T_{2}(\mathbf{e})$, and PD (f) maps, below, were acquired using the Four-FA method [35]. Relaxation time scales (right) are in ms, PD is the fraction relative to water, and green denotes masking 


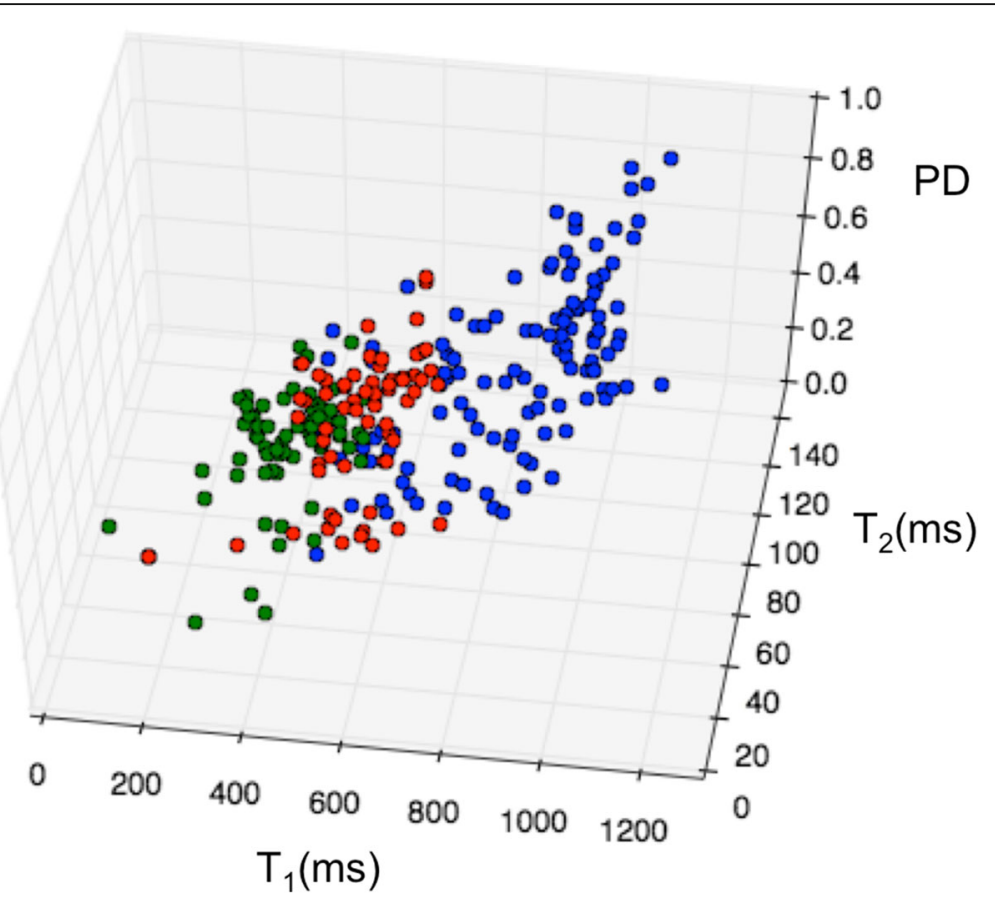

Fig. 3 3D plot of $T_{1}(\mathrm{~ms}), T_{2}$ (ms), and PD (relative to water) values of sampled points from three tissue classes (SMC, blue points; early disease, red; advanced disease, green)

Applying the amplitude and phase corrections from Fig. $1(\mathrm{a}, \mathrm{b})$ to SLAM $\mathrm{T}_{1}, \mathrm{~T}_{2}$ and PD measurements with $R=10$-fold acceleration (effective scan time $=14.3 \mathrm{~s}$ for all three parameters) dramatically improved the accuracy of the larger vessel compartments in Fig. 6(a) (W2, SM, Fat-PD), as compared to no correction (Table 2). These compartments encompass extended portions of the antenna's inhomogeneous sensitivity profile, while in the two smaller lesion compartments (L1, L2), the sensitivity variations are insufficient to corrupt the mean measurements provided by SLAM without the homogeneity corrections. The elongated dSRF of compartment L1 is plotted in Additional file 1.

Post-correction, the SLAM $\mathrm{T}_{1}, \mathrm{~T}_{2}$ and PD parametric maps are consistent with the regular (full $k$-space) MIX images (Fig. 7): the ranges are plotted as a function of $R$ in Fig. 8. For $R \leq 10$, SLAM $\mathrm{T}_{1}, \mathrm{~T}_{2}$ and $\mathrm{PD}$ measurements fall within a standard deviation (SD) for all compartments. The mean values of all parameters in lesion and fat compartments differ from full $k$-space measures by $\leq 0.5 \%( \pm 4 \% \mathrm{SD})$. Even in surrounding tissue and fluid within the vessel where the SDs of the means of $\mathrm{T}_{1}$ and PD from the full $k$-space images are $\geq 30 \%$ (due to low SNR), SLAM $\mathrm{T}_{1}$ and $\mathrm{PD}$ values agreed with the means to within $\leq 6 \%( \pm 6 \% \mathrm{SD}$; data not shown).

In vivo, SLAM reconstruction of six compartments (Fig. 6b; 1, blood; 2, surrounding tissue; 3, artery wall; 4, fat stripe; 5 , vein vessel wall; 6 , 'everything else') also yielded $T_{1}, T_{2}$ and $P D$ values that fell within a SD of the mean compartment values measured from the full $k$ space data, at least up to $R=18$ (Table 3). Note that compartment 6 ('everything else') is a catch-all residual created to meet the criterion that all signal sources be allocated for SLAM reconstruction [31]: it is not meaningful and has a huge SD.

Table 1 Relaxometry and leave-one-out cross-validation results from the SVM automatic lesion classifier vs. histology in autopsied human aortas

\begin{tabular}{llll}
\hline & Smooth muscle & Early disease & Advanced disease \\
\hline $\mathrm{T}_{1}(\mathrm{~ms})$ & $841 \pm 155$ & $561 \pm 104$ & $422 \pm 86$ \\
$\mathrm{~T}_{2}(\mathrm{~ms})$ & $75 \pm 25$ & $53 \pm 23$ & $51 \pm 16$ \\
$\mathrm{PD}$ (vs. water) & $0.68 \pm 0.16$ & $0.63 \pm 0.17$ & $0.54 \pm 0.2$ \\
Histology classification: & 116 & 66 & 66 \\
Correctly classified by SVM: & 96 & 43 & 55 \\
Misclassified by SVM: & 20 & 23 & 11 \\
\hline
\end{tabular}



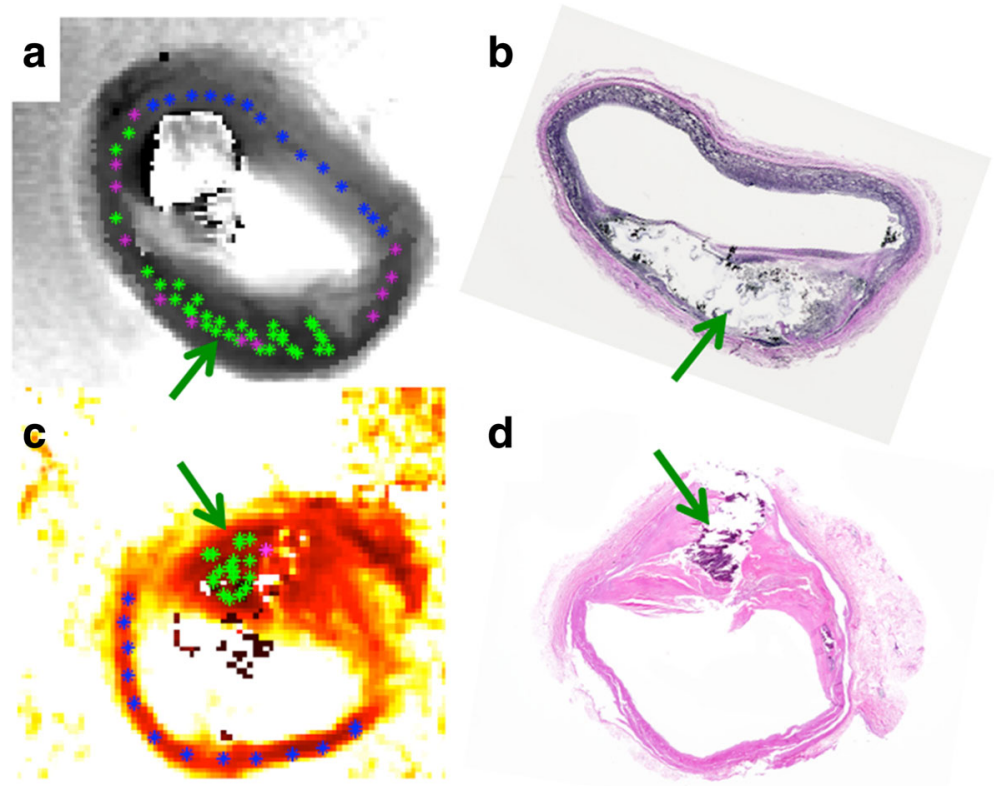

Fig. 4 Application of the SVM classifier to random points in fresh (a, b) and formalin-fixed (c, d) vessel specimens. The points on the $T_{1}$-weighted (a) and true $T_{1}$ (c) images are now color-coded by the classifier as in Fig. 3 (green arrow = advanced lesion). Parts (b) and (d) are WG-stained histological sections (some calcification is lost during staining)

\section{Discussion}

The purpose of this study was two-fold: first, to test whether IVMRI measurements of $\mathrm{T}_{1}, \mathrm{~T}_{2}, \mathrm{PD}$, and lipid imaging could be used to characterize vessel disease in a clinical 3 T scanner; and second, to develop a strategy that could accelerate quantification of these metrics and enable disease characterization in situations where multi-parametric acquisitions are limited by scan-time. To date, the classification of vessel disease into stages IVI using the AHA scale [2] with minimally-invasive X-ray, IVUS and OCT imaging is confounded by poor soft-tissue sensitivity, artifacts and/or the modality's limited depth penetration [3-6]. The characterization of vessel-wall pathology using conventional MRI, which is intrinsically sensitive to soft-tissue, is limited by spatial resolution and long scan-times for $\mathrm{T}_{1}, \mathrm{~T}_{2}$, and PD measurements.

Here, spatial resolution is addressed using IVMRI detectors that offer locally-intense SNR with $\sim 200 \mu \mathrm{m}$ resolution at $\geq 3 \mathrm{~T}[25-27,29]$. Such resolution is not currently achievable with external detectors in clinical scanners. Together with the high vessel-wall SNR afforded by IVMRI, high resolution can avoid the confounding partial-volume effects and signal 'bleed' from adjacent flowing blood. We used a machine-learning SVM classifier trained on histology to automatically differentiate three classes of healthy and atherosclerotic tissue and test the suitability of $\mathrm{T}_{1}, \mathrm{~T}_{2}$ and $\mathrm{PD}$ for classifying vessel disease. The automatic classifier demonstrated an accuracy of $\sim 80 \%$, with an AUC of 0.96 in ROC analysis for disease detection overall (Fig. 5).
Few studies have reported multi-parametric mapping in vessel walls using clinical scanners at $3 \mathrm{~T}$. The use of relaxation-based 'black blood' MRI methods to eliminate contaminating blood signals (e.g. using inversion or saturation) may be a contributing factor as these can confound $\mathrm{T}_{1}$ relaxometry, including that performed with the minimum-acquisition multi-parametric sequences deployed here. Indeed, most vessel wall studies have focused on $\mathrm{T}_{2}$ mapping $[17,21]$ which was believed to better highlight disease [21, 44]. However, except for formalin-fixed tissue, we found that $T_{1}$ betterdiscriminated disease from healthy tissue than $T_{2}$ and $\mathrm{PD}$ alone (Fig. 5), while $\mathrm{T}_{1}$ provided a comparable contrast to $\mathrm{T}_{2}$ for distinguishing early and advanced lesions (Fig. 3, Table 1). In formalin-fixed specimens, the shorter $\mathrm{T}_{2}$ (22 $\mathrm{ms}$ in main text vs $51 \mathrm{~ms}$ in Table 1 ) and lower PD (0.35 vs 0.54 in Table 1$)$ combined with the $\mathrm{T}_{1}$ differences to improve the accuracy of the automatic classifier to near $100 \%$ for distinguishing advanced disease, without any retraining. High $\mathrm{T}_{1}$-contrast was noted for vessel wall components measured at 9.4 $\mathrm{T}$ in the only other report we found with both $\mathrm{T}_{1}$ and $\mathrm{T}_{2}$ data from atherosclerotic tissue [45]. However, as evident from the published values listed in 'Results', prior vascular relaxation measurements lack consensus, which may reflect the spatial resolution, SNR, and scan-time limitations of conventional approaches. Even so, our measured $T_{1}$ and $T_{2}$ values lie within the range of previous results [17, 19-22].

To our knowledge, this study is the first to combine multi-parametric MRI mapping with an advanced 

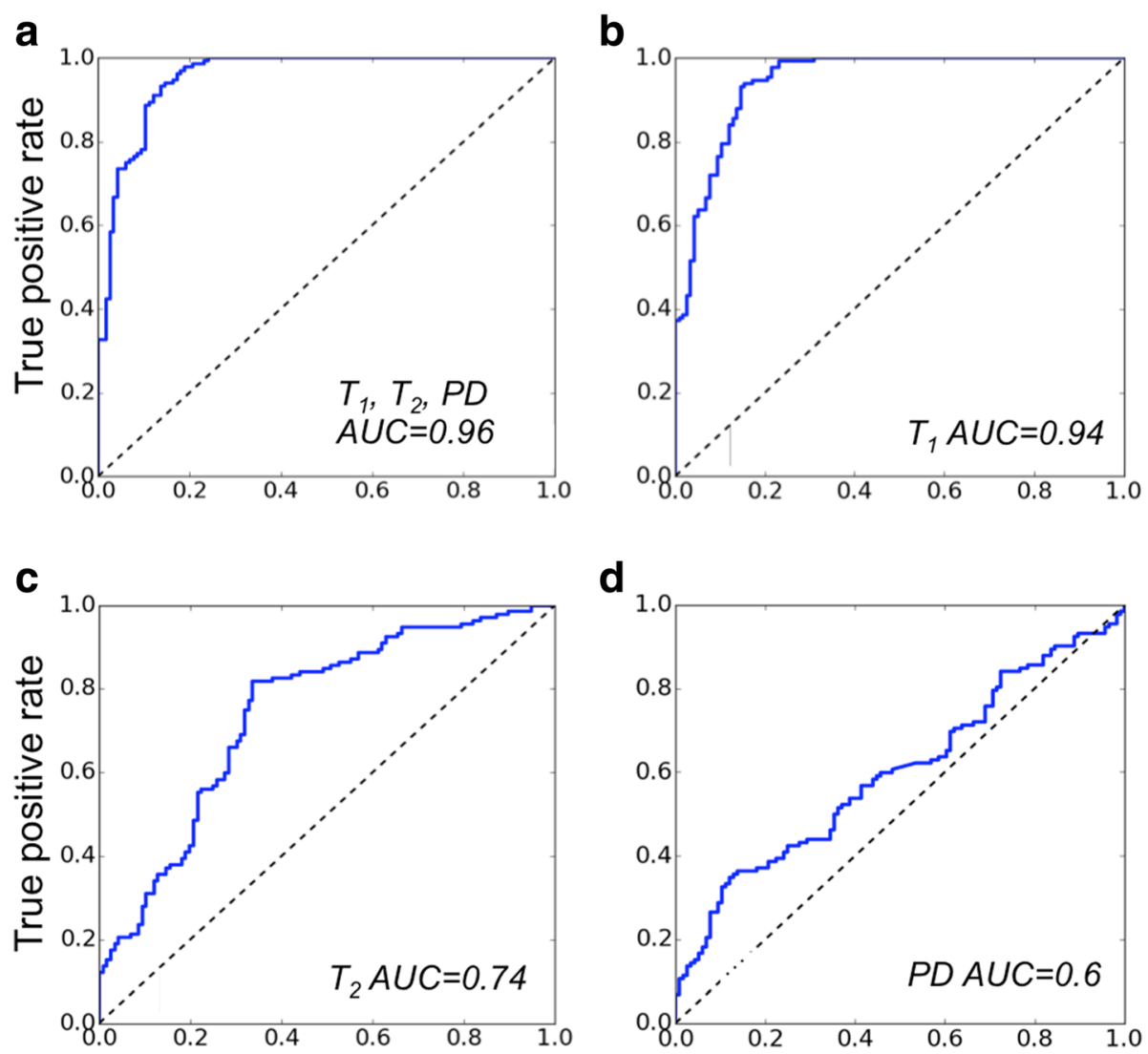

False positive rate

d

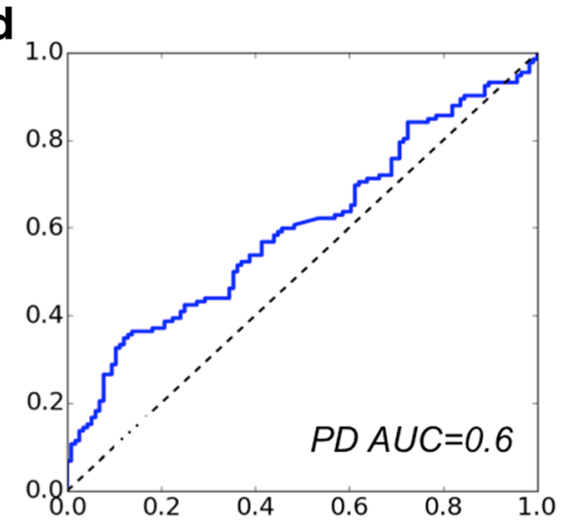

False positive rate

Fig. 5 ROC curves of the classifier for distinguishing diseased and healthy tissue based on: (a) all three parameters, $T_{1}, T_{2}$ and $P D ;(\mathbf{b}) T_{1}$ only, (c) $\mathrm{T}_{2}$, only and (d) PD only. Areas under the curves (AUCs) are $0.96,0.94,0.74$ and 0.6 respectively
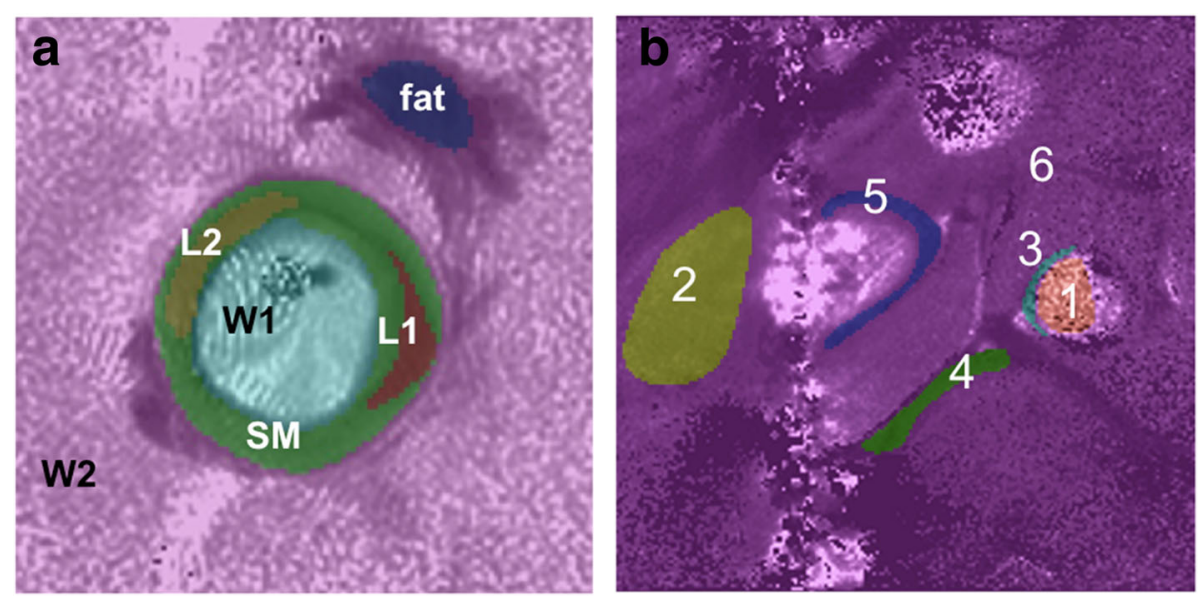

Fig. 6 (a) Six anatomical compartments segmented for SLAM reconstruction overlaid on a $T_{1}$-weighted IV image from a diseased vessel specimen (fat; two lesions, L1 and L2; vessel fluid contents, W1; smooth vessel-wall muscle, SM; surrounding tissue $=$ W2). (b) In vivo SLAM segmentation of six compartments on an in vivo $T_{1}$ image of the inferior vena cava (1, blood; 2 , surrounding tissue; 3 , arterial wall; 4, fat stripe; 5 , vein wall; 6 , everything else) 
Table 2 Effect of magnitude and phase corrections on SLAM relaxation and $P D$ measurements with $R=10$-fold acceleration

\begin{tabular}{llll}
\hline & No correction & With Correction & True value $^{\mathrm{a}}$ \\
\hline W2 $_{1}$ (ms) & 3700 & 2780 & $2700 \pm 800$ \\
W2 $_{2}$ (ms) & $-15,240$ & 1320 & $1200 \pm 1380$ \\
SM PD (vs. water) & 0.2 & 0.83 & $0.82 \pm 0.2$ \\
Fat $T_{1}$ (ms) & 370 & 373 & $370 \pm 100$ \\
Fat $T_{2}$ (ms) & 104 & 104 & $112 \pm 31$ \\
Fat PD (vs. water) & 0.44 & 0.7 & $0.67 \pm 0.11$ \\
L1 $T_{1}$ (ms) & 493.4 & 507 & $514 \pm 98$ \\
L1 T $_{2}$ (ms) & 52 & 51.7 & $53.4 \pm 7.5$ \\
L1 PD (vs. water) & 0.89 & 0.89 & $0.84 \pm 0.09$ \\
L2 T $T_{1}$ (ms) & 509.5 & 490.5 & $475 \pm 127$ \\
L2 $T_{2}$ (ms) & 70 & 69 & $67.2 \pm 16$ \\
L2 PD (vs. water) & 0.67 & 0.63 & $0.64 \pm 0.12$ \\
\hline
\end{tabular}

${ }^{a}$ Mean \pm SD for each compartment defined in Fig. 6(a) as measured from the full $k$-space data sets. $S M=$ smooth vessel-wall muscle; $W 2$ = surrounding tissue; $\mathrm{L} 1$ = lesion $1 ; \mathrm{L} 2$ = lesion 2 machine learning algorithm to classify plaques. However, plaque characterization using only $\mathrm{T}_{2}$ and a fuzzy ' $\mathrm{C}$ means' classifier has been reported [46]. We found that the combination of all three parameters, $\mathrm{T}_{1}, \mathrm{~T}_{2}$ and $\mathrm{PD}$, provided the most accurate disease classification in $3 \mathrm{D}$ 'feature space' (Table 1, Figs. 3 and 5). The SVM approach maps the parametric data to a high-dimensional space to compute a hyper-plane that optimizes the separation of the training-dataset into the designated classes. The three-class SVM classifier was robust to varying sample conditions as evidenced by its ability to correctly classify tissue in refrigerated, formalin-fixed and fresh specimens (Fig. 4), as well as healthy in vivo tissue after training on fresh specimens alone. The SVM partitions the 3D space with decision planes such that the vectors normal to the planes are in directions that maximally separate data points from the two adjacent classes. Therefore, weighted images acquired with sequence parameters that correspond to the normal direction of
Full $k$-space
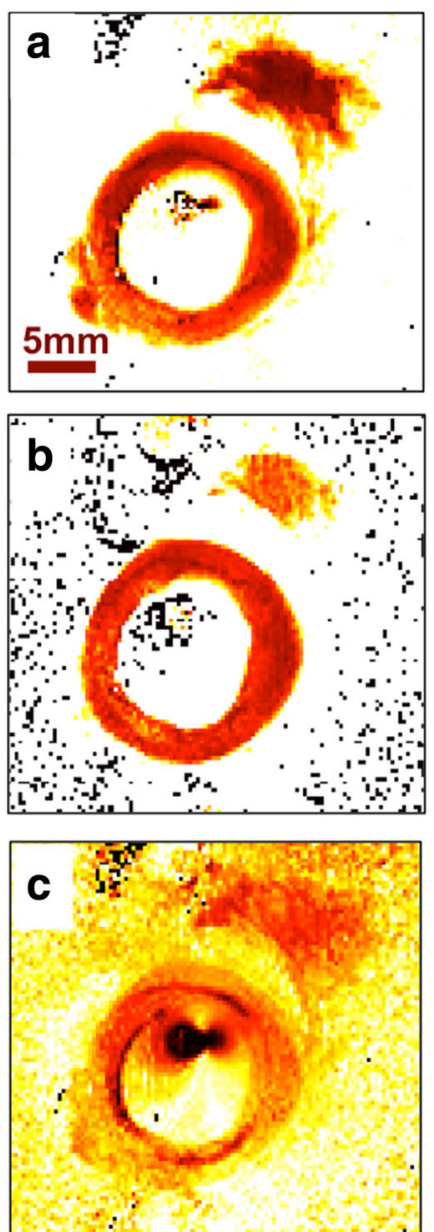

$10 \%$ k-space (SLAM)
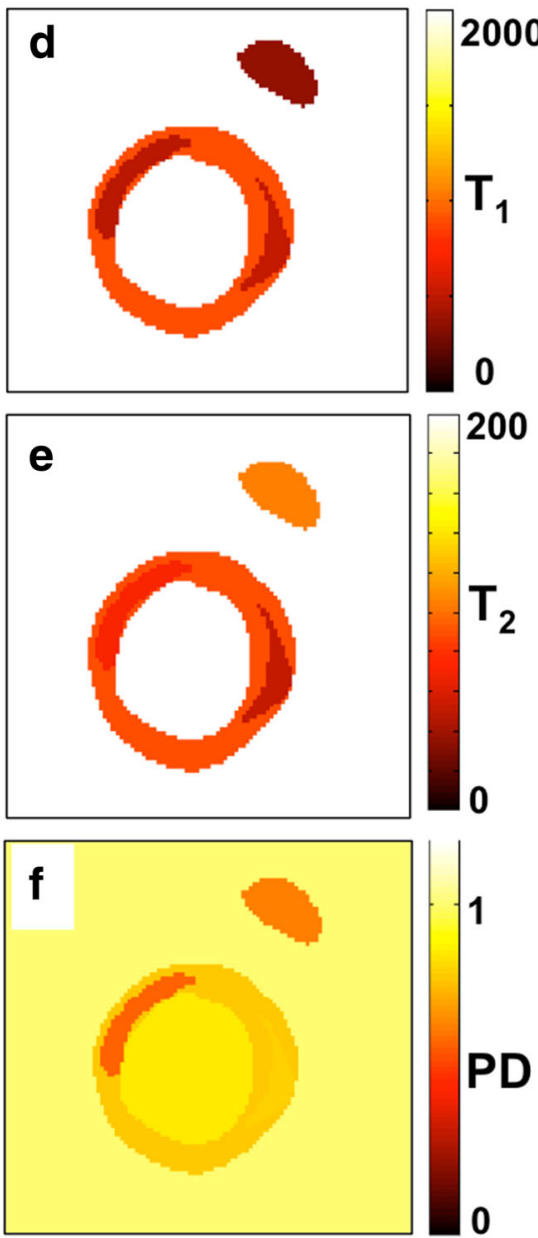

Fig. 7 Color-coded $T_{1}(\mathrm{~ms} ; \mathbf{a}, \mathbf{d}), T_{2}(\mathrm{~ms} ; \mathbf{b}, \mathbf{e})$ and PD (=1 for water; $\left.\mathbf{c}, \mathbf{f}\right)$ maps calculated using the full $k$-space MIX-TSE data (a-c); and with SLAM using only $10 \%$ of the central $k$-space data $(\mathbf{d}-\mathbf{f})$ and the magnitude and phase corrections shown in Fig. $1(\mathrm{a}-\mathrm{b})$ 


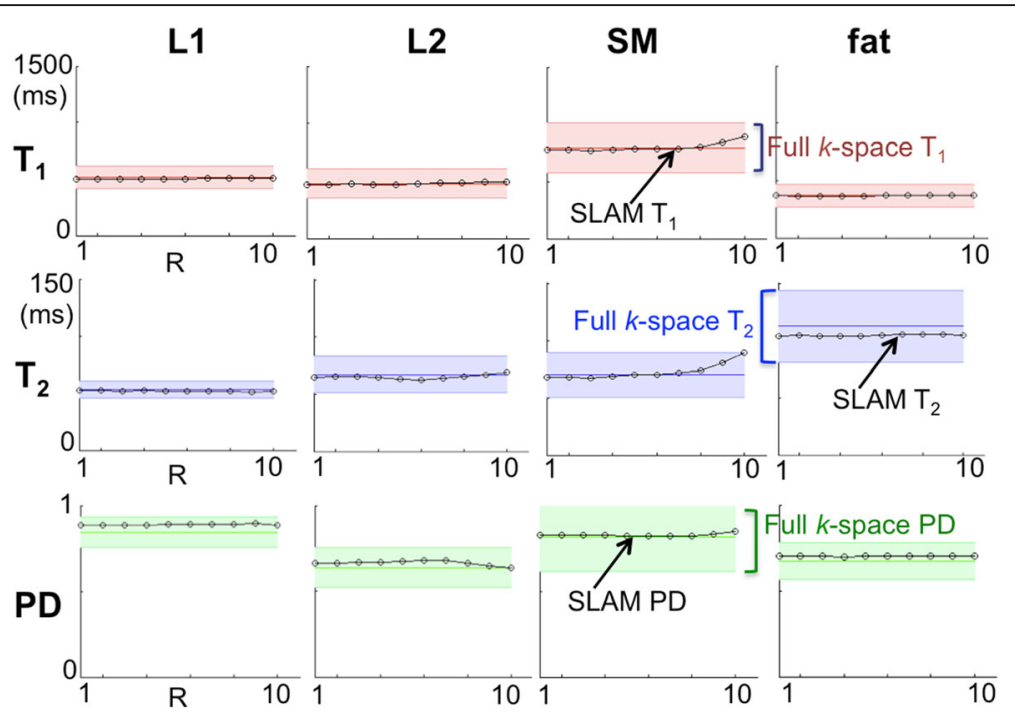

Fig. $8 \mathrm{SLAM} T_{1}, T_{2}$ and PD values from four compartments of the autopsy specimen shown in Fig. 3(a) as a function of $R$. Colored error bands denote compartment mean values \pm SD as derived from the corresponding full $k$-space images. SLAM and full $k$-space measures of $T_{1}, T_{2}$ and PD in fluid and surrounding tissue compartments (not shown) also agree

Table 3 Comparison of in vivo vessel compartment-average $T_{1}$ (ms), $T_{2}$ (ms), and PD (relative to water) measured using SLAM with acceleration factors of $R=1-18$, and those measured using the full $k$-space reconstruction

\begin{tabular}{llllllll}
\hline & Comp. $^{a}$ & $\mathrm{R}=1$ & $\mathrm{R}=3$ & $\mathrm{R}=6$ & $\mathrm{R}=10$ & $\mathrm{R}=18$ & full -space \\
\hline $\mathrm{T}_{1}$ & 1 & 2916 & 2905 & 2900 & 2946 & 2826 & $2802 \pm 721$ \\
& 2 & 787 & 787 & 788 & 786 & 780 & $812 \pm 124$ \\
& 3 & 848 & 865 & 993 & 1094 & 1041 & $929 \pm 388$ \\
& 4 & 236 & 237 & 243 & 288 & 365 & $253 \pm 122$ \\
& 5 & 731 & 728 & 740 & 804 & 879 & $776 \pm 123$ \\
& 6 & 705 & 705 & 704 & 705 & 704 & $964 \pm 704$ \\
$\mathrm{~T}_{2}$ & 1 & 1173 & 1154 & 1131 & 1040 & 825 & $1050 \pm 1253$ \\
& 2 & 47 & 47 & 47 & 47 & 47 & $51 \pm 15$ \\
& 3 & 75 & 81 & 106 & 123 & 135 & $128 \pm 247$ \\
& 4 & 99 & 99 & 98 & 93 & 86 & $98 \pm 21.5$ \\
& 5 & 42 & 42 & 42 & 43 & 47 & $46 \pm 10$ \\
6 & 64 & 64 & 64 & 63 & 64 & $101 \pm 176$ \\
$\mathrm{PD}$ & 1 & 1.01 & 1.01 & 1.00 & 1.00 & 0.95 & $1.00 \pm 0.24$ \\
& 2 & 0.77 & 0.77 & 0.78 & 0.78 & 0.77 & $0.74 \pm 0.13$ \\
3 & 0.45 & 0.44 & 0.45 & 0.50 & 0.52 & $0.46 \pm 0.18$ \\
4 & 0.41 & 0.40 & 0.40 & 0.39 & 0.38 & $0.45 \pm 0.10$ \\
5 & 0.81 & 0.81 & 0.82 & 0.84 & 0.83 & $0.84 \pm 0.18$ \\
6 & 0.40 & 0.40 & 0.40 & 0.40 & 0.40 & $0.65 \pm 0.54$ \\
\hline
\end{tabular}

${ }^{a}$ Compartments as defined in Fig. 6(b)

${ }^{\mathrm{b}}$ Mean $\pm \mathrm{SD}$ of compartment the decision plane should on average provide the maximum contrast between two adjacent classes.

A prior study at $1.5 \mathrm{~T}$ reported that qualitative assessments of lesion appearance in time-of-flight, $\mathrm{T}_{1^{-}}$and $\mathrm{T}_{2^{-}}$ weighted MRI could be used for AHA disease classification [14]. Our studies indicate that $\mathrm{T}_{1}, \mathrm{~T}_{2}$, and PD may suffice to discriminate atherosclerotic lesion stage, and that a three-parameter SVM classifier could do so automatically. The SVM might identify additional AHA classifiers or features [2] as well, but the present study was constrained by the availability of fresh human vessel specimens exhibiting a sufficient number of histologically diverse pathologies needed for training. Note that training the SVM classifier is cumulative: adding more data from further studies will better tune its accuracy and define its potential for quantitative IVMRI disease classification. Although our data sampled specimens from a limited group of decedents, the training of machine-learning classifiers does not require independent training samples [47], and the versatility of our trained SVM classifier was demonstrated by its success with formalin-fixed specimens. With enough pathology samples a plaque component classifier might ultimately be able to automatically identify calcifications (stage V), which appear as signal voids in scout and endoscopic images [25]; fibrous caps (stages II-III) which are measurable by IVMRI [29]; lipids in AHA class I-III lesions revealed by Dixon imaging (Fig. 2a); and perhaps even vulnerable plaque to the extent it is identifiable in training specimens and distinguishable via its IVMRI parameter set. 
Our strategy to speed-up the acquisition of multiparametric $\mathrm{T}_{1}, \mathrm{~T}_{2}$, and $\mathrm{PD}$ mapping by employing sensitivity-weighted SLAM reconstruction [30, 31], yielded compartment-average measurements up to $R=18$ times faster than regular IVMRI. This makes an extraordinary dent on the long scan-times conventionally required for parametric mapping, and reduces opportunities for physiological motion to corrupt data in vivo. A virtue of SLAM is that the compartments which are segmented from highresolution scout scans, can be re-adjusted at will from the same data set post-acquisition [34]. In principle, the acceleration factor, $R$, for SLAM reconstruction can be increased by reducing the number of phase-encoding steps until it equals the number of compartments: the maximum is $R=30$ for Fig. 6 , for example. By encoding compartments directly using the highest-SNR spatial encoding steps, SLAM realizes an SNR gain roughly proportional to the square-root of the number of voxels in the compartment, as compared to summing the same voxel signals from the high-resolution image, post-acquisition [30-34]. This partially offsets the SNR lost by reducing the acquisition time. In contrast, the application of compressedSENSE undersampling to IVMRI at $3 \mathrm{~T}$ using the same nominal spatial resolution, realizes no volume SNR gain that could offset the SNR loss from acceleration, and acceleration has been limited to $R \leq 4$ [40].

SLAM assumes a uniform signal within each compartment wherein the reconstruction is an exact solution of a set of simultaneous equations (see Additional file 1) $[30,31]$. While as few as one voxel can be assigned to a compartment from the segmentation image, an SNR penalty proportional to the compartment volume is paid as compartments are shrunk. Deviations from the uniformity assumption can introduce bleed error [30], which is ameliorated using the 'SLAM2' algorithm [30, 31, 34]. Here, simple linear $0-2 \pi$ phase and $r$-corrections adequately addressed the loopless antenna's highly nonuniform sensitivity profile, as evidenced by SLAM's accuracy up to $R=10$ (Fig. 8). Owing to the device's cylindrical symmetry [27], the correction is relatively robust to antenna orientations askew from the $\mathrm{B}_{0}$-axis, although such corrections may not suffice for larger $R$ values, especially for larger compartments encompassing larger areas of nonuniformity. Nevertheless, SLAM offers dramatic reductions in scantimes allowing quantitative multi-parametric IVMRI in $\leq 15 \mathrm{~s}$, even in regions of low SNR.

\section{Conclusion}

High-resolution, sensitivity-corrected, multi-parametric IVMRI in combination with a supervised machine learning SVM classifier can automatically classify normal, early and advanced atherosclerosis with $\sim 80 \%$ accuracy and an AUC of $\sim 0.96$ based on $\mathrm{T}_{1}, \mathrm{~T}_{2}$ and PD measurements. Replacing IVMRI relaxometry with sensitivity-corrected intravascular SLAM relaxometry provides equivalent estimates of $T_{1}, T_{2}$ and PD an order-of-magnitude faster to facilitate IVMRI-based characterization of vessel disease.

A possible modus operandi for this technology is to perform high-resolution $3 \mathrm{~T}$ scout IVMRI using a regular gradient-echo or steady-state free-precession sequence with MRI endoscopy at 1-2 fr/s [25], or another high-speed undersampling method [40]. At a region of suspected disease, the sequence would be switched to a $\mathrm{T}_{1} / \mathrm{T}_{2}$ acquisition employing a Four-FA [35], MIX-TSE or other high-speed relaxometry method; but with all of the phase-encode steps omitted save for, say, 6-10 steps lying at the center of image $k$-space to provide an order(s)-of-magnitude speed-up. Post-acquisition, 6-10 compartments of interest would be segmented from the regular IVMRI, and their $\mathrm{T}_{1}, \mathrm{~T}_{2}$ and $\mathrm{PD}$ values reconstructed using the sensitivity-corrected SLAM algorithm presented herein. Vessel disease could then be characterized automatically with the SVM classifier.

\section{Additional file}

Additional file 1: Supplementary file. (PDF $2140 \mathrm{~kb}$ )

\section{Abbreviations}

2D: Two-dimensional; 3D: Three-dimensional; AHA: American Heart Association; AUC: Area under curve; BIR4: $\mathrm{B}_{1}$-independent rotation; FA: flip-angle; Four-

FA: Four flip-angle; H\&E: Hematoxylin and eosin; IV: Intravascular; IVUS: Intravascular ultrasound; MIX-TSE: Mixed turbo spin-echo; MRI: Magnetic resonance imaging; OCT: Optical coherence tomography; OD: Outer diameter; PD: Proton density; ROC: Receiver operating characteristic; SD: Standard deviation; SENSE: Sensitivity encoding; SLAM: Spectroscopy with linear algebraic modeling; SMC: Smooth muscle cells; SNR: Signal-to-noise ratio; SVM: Support vector machine; $T_{1}$ : Spin-lattice relaxation time; $T_{2}$ : Spin-spin relaxation time; TE: Echo time; TI: inversion time; TR: sequence repetition period; WG: Verhoeffvan Gieson

\section{Acknowledgements}

We thank Dara L. Kraitchman, Parag Karmarkar and Xiaoyang Liu for their assistance with the in vivo study.

\section{Funding}

This work was supported by Supported by NIH grant R01 EB007829.

\section{Availability of data and materials}

Data generated or analyzed during this study are included in this published article and its supplementary information files.

\section{Authors' contributions}

GW was responsible for designing and implementing experimental studies, SVM analysis, interpreting the data, and preparing the initial manuscript. YZ modified the SLAM reconstruction for IVMRI, analyzed SLAM data and assisted with experiments and manuscript preparation. SSH assisted with probe fabrication, study design, experiments and manuscript preparation. PAB was responsible for study conception, design, interpretation, critical review and drafting the final manuscript. All authors read and approved the final manuscript.

\section{Ethics approval and consent to participate}

The in vitro study of tissue from decedents was obtained from our Pathology Department with approval from the Johns Hopkins Institutional Review Board, and did not require additional consent from participants or their living representatives. The in vivo animal study was approved by Institutional Animal Care and Use Committee. 


\section{Consent for publication}

Not applicable.

\section{Competing interests}

The authors declare that they have no competing interests.

\section{Publisher's Note}

Springer Nature remains neutral with regard to jurisdictional claims in published maps and institutional affiliations.

Received: 16 June 2017 Accepted: 16 October 2017 Published online: 20 November 2017

\section{References}

1. Mozaffarian D, Benjamin EJ, Go AS, Arnett DK, Blaha MJ, Cushman M, Das SR, de Ferranti S, Després J-P, Fullerton HJ, et al. Heart disease and stroke statistics-2016 update. Circulation. 2016;133:e38-e360.

2. Stary HC, Chandler AB, Dinsmore RE, Fuster V, Glagov S, Insull W, Rosenfeld ME, Schwartz CJ, Wagner WD, Wissler RW. A definition of advanced types of atherosclerotic lesions and a histological classification of atherosclerosis a report from the committee on vascular lesions of the council on arteriosclerosis, American Heart Association. Circulation. 1995;92:1355-74.

3. Fuster V, Fayad ZA, Moreno PR, Poon M, Corti R, Badimon JJ. Atherothrombosis and high-risk plaque: part II: approaches by noninvasive computed tomographic/magnetic resonance imaging. J Am Coll Cardiol. 2005:46:1209-18.

4. Guagliumi G, Virmani R. The race to achieve the gold standard in coronary imaging. Rev Esp Cardiol. 2009;62:599-602.

5. Meissner OA, Rieger J, Rieber J, Klauss V, Siebert U, Tato F, Pfeifer K, Reiser $M$, Hoffmann U. High-resolution MR imaging of human atherosclerotic femoral arteries in vivo: validation with intravascular ultrasound. J Vasc Interv Radiol. 2003;14:227-31.

6. Devuyst G, Karapanayiotides T, Ruchat P, Pusztaszeri M, Lobrinus JA, Jonasson L, Cuisinaire O, Kalangos A, Despland PA, Thiran JP, Bogousslavsky J. Ultrasound measurement of the fibrous cap in symptomatic and asymptomatic atheromatous carotid plaques. Circulation. 2005;111:2776-82.

7. Sanz J, Fayad ZA. Imaging of atherosclerotic cardiovascular disease. Nature. 2008:451:953.

8. Tarkin JM, Dweck MR, Evans NR, Takx RA, Brown AJ, Tawakol A, Fayad ZA, Rudd JH. Imaging atherosclerosis. Circ Res. 2016;1 18:750-69.

9. W-H X, Li M-L, Gao S, Ni J, Zhou L-X, Yao M, Peng B, Feng F, Jin Z-Y, Cui L-Y. In vivo high-resolution $M R$ imaging of symptomatic and asymptomatic middle cerebral artery atherosclerotic stenosis. Atherosclerosis. 2010;212:507-11.

10. Ryu CW, Jahng GH, Kim EJ, Choi WS, Yang DM. High resolution wall and lumen MRI of the middle cerebral arteries at 3 tesla. Cerebrovasc Dis. 2009; 27:433-42.

11. Coolen BF, Calcagno C, van Ooij P, Fayad ZA, Strijkers GJ, Nederveen AJ. Vessel wall characterization using quantitative MRI: what's in a number? New York: Magma; 2017.

12. Saam T, Hetterich H, Hoffmann V, Yuan C, Dichgans M, Poppert H, Koeppel T, Hoffmann U, Reiser MF, Bamberg F. Meta-analysis and systematic review of the predictive value of carotid plaque hemorrhage on cerebrovascular events by magnetic resonance imaging. J Am Coll Cardiol. 2013;62:1081-91.

13. Touboul PJ, Elbaz A, Koller C, Lucas C, Adrai V, Chedru F, Amarenco P. Common carotid artery intima-media thickness and brain infarction : the etude du Profil Genetique de l'Infarctus cerebral (GENIC) case-control study. The GENIC investigators. Circulation. 2000;102:313-8.

14. Cai J-M, Hatsukami TS, Ferguson MS, Small R, Polissar NL, Yuan C. Classification of human carotid atherosclerotic lesions with in vivo multicontrast magnetic resonance imaging. Circulation. 2002;106:1368-73.

15. Saam T, Ferguson MS, Yarnykh VL, Takaya N, Xu D, Polissar NL, Hatsukami TS, Yuan C. Quantitative evaluation of carotid plaque composition by in vivo MRI. Arterioscler Thromb Vasc Biol. 2005;25:234-9.

16. Bottomley PA, Hardy CJ, Argersinger RE, Allen-Moore G. A review of $1 \mathrm{H}$ nuclear magnetic resonance relaxation in pathology: are $\mathrm{T} 1$ and $\mathrm{T} 2$ diagnostic? Med Phys. 1987;14:1-37.

17. Biasiolli L, Lindsay A, Chai J, Choudhury R, Robson M. In-vivo quantitative T2 mapping of carotid arteries in atherosclerotic patients: segmentation and T2 measurement of plaque components. J Cardiovasc Magn Reson. 2013;15:69.

18. Rogers WJ, Prichard JW, Y-L H, Olson PR, Benckart DH, Kramer CM, Vido DA, Reichek N. Characterization of signal properties in atherosclerotic plaque components by intravascular MRI. Arterioscler Thromb Vasc Biol. 2000;20: 1824-30.

19. Dalager-Pedersen S, Falk E, Ringgaard S, Kristensen IB, Pedersen EM. Effects of temperature and histopathologic preparation on the size and morphology of atherosclerotic carotid arteries as imaged by MRI. J Magn Reson Imaging. 1999;10:876-85.

20. Coolen BF, Poot DH, Liem MI, Smits LP, Gao S, Kotek G, Klein S, Nederveen AJ. Three-dimensional quantitative $\mathrm{T} 1$ and $\mathrm{T} 2$ mapping of the carotid artery: sequence design and in vivo feasibility. Magn Reson Med. 2016;75(3):100817. doi:10.1002/mrm.25634.

21. Raynaud J-S, Bridal SL, Toussaint J-F, Fornès $P$, Lebon V, Berger $G$, LeroyWillig A. Characterization of atherosclerotic plaque components by high resolution quantitative MR and US imaging. J Magn Reson Imaging. 1998;8: 622-9.

22. Mihai G, Giri S, Sharkey-Toppen T, Raman S, Rajagopalan S, Simonetti O. Quantitative $\mathrm{T} 1, \mathrm{~T} 2$ and $\mathrm{T} 2^{*}$ mapping of carotid artery normal wall and atherosclerotic plaque. In: Proc Int Soc Magn Reson Med; 2011. p. 3311.

23. Martin AJ, Plewes DB, Henkelman RM. MR imaging of blood vessels with an intravascular coil. J Magn Reson Imaging. 1992;2:421-9.

24. Larose E, Yeghiazarians Y, Libby P, Yucel EK, Aikawa M, Kacher DF, Aikawa E, Kinlay S, Schoen FJ, Selwyn AP, Ganz P. Characterization of human atherosclerotic plaques by intravascular magnetic resonance imaging. Circulation. 2005;112:2324-31.

25. Sathyanarayana S, Schär M, Kraitchman DL, Bottomley PA. Towards real-time intravascular endoscopic magnetic resonance imaging. J Am Coll Cardiol Img. 2010;3:1158-65.

26. Ertürk MA, El-Sharkawy A-MM, Bottomley PA. The interventional Loopless antenna at 7 Tesla. Magn Reson Med. 2012;68:980-8.

27. Sathyanarayana S, Bottomley PA. MRI endoscopy using intrinsically localized probes. Med Phys. 2009;36:908-19.

28. El-Sharkawy AM, Qian D, Bottomley PA. The performance of interventional loopless MRI antennae at higher magnetic field strengths. Med Phys. 2008; 35:1995-2006.

29. Qian D, Bottomley PA. High-resolution intravascular magnetic resonance quantification of atherosclerotic plaque at 3T. J Cardiovasc Magn Reson. 2012;14:20.

30. Zhang Y, Gabr RE, Schär M, Weiss RG, Bottomley PA. Magnetic resonance spectroscopy with linear algebraic modeling (SLAM) for higher speed and sensitivity. J Magn Reson. 2012;218:66-76.

31. Zhang Y, Gabr RE, Zhou J, Weiss RG, Bottomley PA. Highly-accelerated quantitative $2 \mathrm{D}$ and $3 \mathrm{D}$ localized spectroscopy with linear algebraic modeling (SLAM) and sensitivity encoding. J Magn Reson. 2013;237:125-38.

32. Zhang Y, Heo HY, Jiang S, Lee DH, Bottomley PA, Zhou J. Highly accelerated chemical exchange saturation transfer (CEST) measurements with linear algebraic modeling. Magn Reson Med. 2016;76:136-44.

33. Bottomley PA, Zhang Y. Accelerated Spatially Encoded Spectroscopy of Arbitrarily Shaped CompartmentsUsing Prior Knowledge and Linear Algebraic Modeling. eMagRes. 2015;4:89-104. doi:10.1002/9780470034590. emrstm1438.

34. Zhang Y, Liu X, Zhou J, Bottomley PA. Ultrafast compartmentalized relaxation time mapping with linear algebraic modeling. Magn Reson Med. 2017;

35. Wang G, El-Sharkawy A-MM, Bottomley PA. Minimum acquisition methods for simultaneously imaging $\mathrm{T} 1, \mathrm{~T} 2$, and proton density with $\mathrm{B} 1$ correction and no spin-echoes. J Magn Reson. 2014;242:243-55.

36. Jensen $\mathrm{M}$, Caruthers $\mathrm{S}$, Jara $\mathrm{H}$. Quantitative magnetic resonance imaging with the mixed turbo spin-echo pulse sequence: a validation study. Internet J Radiol. 2001;2(1).

37. Cortes C, Vapnik V. Support-vector networks. Mach Learn. 1995;20:273-97.

38. Suykens JA, Vandewalle J. Least squares support vector machine classifiers. Neural Process Lett. 1999;9:293-300.

39. Glover GH, Schneider E. Three-point dixon technique for true water/fat decomposition with B0 inhomogeneity correction. Magn Reson Med. 1991; 18:371-83.

40. Hegde SS, Zhang Y, Bottomley PA. Acceleration and motion-correction techniques for high-resolution intravascular MRI. Magn Reson Med. 2015;74:452-61.

41. Cuppen J. RLSQ: T1, T2, and $\rho$ calculations, combining ratios and least squares. Magn Reson Med. 1987;5:513-24.

42. Li K-C. Asymptotic optimality for $C_{p}, C_{L}$, cross-validation and generalized cross-validation: discrete index set. Ann Stat. 1987;15:958-75.

43. Hanley JA. Receiver operating characteristic (ROC) methodology: the state of the art. Crit Rev Diagn Imaging. 1989;29:307-35. 
44. Martin AJ, Gotlieb Al, Henkelman RM. High-resolution MR imaging of human arteries. J Magn Reson Imaging. 1995;5:93-100.

45. Morrisett J, Vick W, Sharma R, Lawrie G, Reardon M, Ezell E, Schwartz J, Hunter G, Gorenstein D. Discrimination of components in atherosclerotic plaques from human carotid endarterectomy specimens by magnetic resonance imaging ex vivo. Magn Reson Imaging. 2003;21:465-74.

46. Sun B, Giddens DP, Long R, Taylor WR, Weiss D, Joseph G, Vega D, Oshinski $\mathrm{JN}$. Automatic plaque characterization employing quantitative and multicontrast MRI. Magn Reson Med. 2008;59:174-80.

47. Dundar M, Krishnapuram B, Bi J, Rao RB. Learning classifiers when the training data ss Not IID. In: Proceedings of the International Joint Conference on Artifical Intelligence. 2007. p. 756-761. Available from http://www.jicai.org/Proceedings/07/Papers/121.pdf.

Submit your next manuscript to BioMed Central and we will help you at every step:

- We accept pre-submission inquiries

- Our selector tool helps you to find the most relevant journal

- We provide round the clock customer support

- Convenient online submission

- Thorough peer review

- Inclusion in PubMed and all major indexing services

- Maximum visibility for your research

Submit your manuscript at www.biomedcentral.com/submit
Biomed Central 